\title{
¿Es compatible la durabilidad con la sostenibilidad en la industria de la construcción?
}

\author{
P. Garcés ${ }^{1}$, E. Zornoza ${ }^{1}$, F. Baeza ${ }^{1}$, O. Galao ${ }^{1}$, J. Payá².
}

${ }^{1}$ Departamento de Ingeniería de la Construcción, Universidad de Alicante, España.

${ }^{2}$ Instituto de Ciencia y Tecnología del Hormigón (ICITECH). Universidad Politécnica de Valencia, España.

\begin{abstract}
Información del artículo
DOI:

http://dx.doi.org/10.21041/ra.v2

$\underline{i 2.27}$

Artículo recibido el 03 de

Febrero de 2012, revisado bajo

las políticas de publicación de

la Revista ALCONPAT y

aceptado el 20 de Abril de 2012.

Cualquier discusión, incluyendo

la réplica de los autores se publicará en el primer número del año 2013 siempre y cuando la información se reciba antes del cierre del tercer número del año 2012
\end{abstract}

(C) 2012 ALCONPAT Internacional

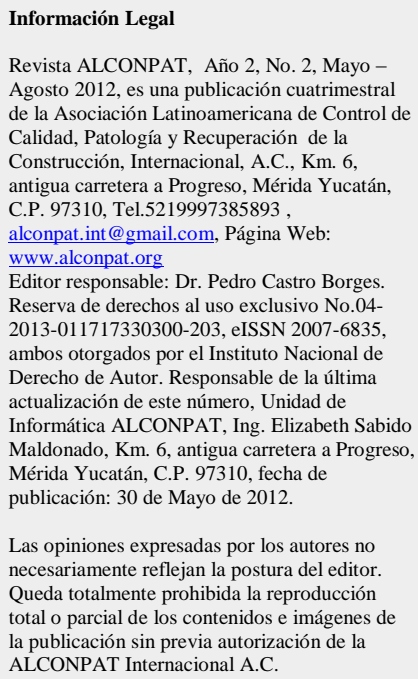

\section{RESUMEN}

En este artículo se presentan resultados obtenidos en distintas propiedades relacionadas con la durabilidad y características resistentes de morteros de cemento parcialmente sustituidos con diferentes residuos agrícola e industriales que presentan actividad puzolánica: ceniza de lodo de depuradora (CLD), ceniza volante (CV), residuo de catalizador de craqueo catalítico (FCC), ceniza de cascara de arroz (CCA), reforzados con acero. Los resultados presentados demuestran que es posible un desarrollo sostenible de la industria de la construcción. Esto puede conseguirse mediante la reducción del contenido de clinker en los cementos y reutilizando subproductos industriales y agrícolas con un adecuado nivel de durabilidad de los materiales de construcción elaborados a partir de ellos.

Palabras claves: durabilidad; sostenibilidad; subproductos industriales; subproductos agrícolas.

\section{ABSTRACT}

This paper presents the results obtained in the evaluation of a series of properties related to durability and strength of steel reinforced cement mortars partially substituted with different agricultural and industrial residues presenting pozzolanic activity: sewage sludge ash (SSA), fly ash (FA), spent catalytic cracking catalyst (FCC) and rice husk ash (RHA). These results show that it is possible to achieve a sustainable development in the construction industry. This can be obtained by reducing the clinker content in cement and reusing industrial and agricultural byproducts with an appropriate level of durability of the construction materials fabricated from them.

Keywords: durability; sustainability; industrial byproduct; agricultural byproduct.

Autor de contacto: Pedro Garcés Terradillos (pedro.garces@ua.es) 
Revista ALCONPAT, Volumen 2, Número 2, Mayo - Agosto 2012, Páginas 57 - 71

\section{INTRODUCCION}

La utilización del hormigón como material de construcción es una práctica muy extendida en todo el mundo, lo cual puede ser explicado por su elevada resistencia mecánica, versatilidad de su encofrado y por su coste relativamente bajo. El hormigón es el material industrial más utilizado en la construcción civil y el segundo más consumido en el mundo, siendo superado únicamente por el agua. La producción de hormigón está asociada habitualmente con el uso del cemento Pórtland, por ser éste el principal conglomerante utilizado en la elaboración de morteros y hormigones. En el año 2008 la producción mundial de cemento Pórtland superó los 2600 millones de toneladas, lo que supone un consumo aproximado de $1 \mathrm{~m}^{3}$ de hormigón por habitante. Las estimaciones señalan que, en el año 2050, el consumo de cemento Pórtland podría incrementarse hasta un $225 \%$ con respecto a los valores actuales, debido principalmente a la elevada demanda de este conglomerante por parte de países emergentes, como pueden ser India, China, Brasil y México.

No obstante, no se debe perder de vista que la producción de cemento Pórtland requiere un elevado consumo de energía y de materias primas no renovables. Además, genera grandes cantidades de desechos y gases de efecto invernadero que contaminan el medio ambiente, lo que perjudica la armonía entre el ser humano y la naturaleza. Esta degradación medioambiental no es un problema reciente, ni tampoco un problema exclusivo de un determinado país o región. Se trata de un problema global, asociado al crecimiento poblacional y a la urbanización del planeta. La consecuencia negativa de este crecimiento exagerado es el aumento en la producción de agentes contaminantes que contribuyen al calentamiento global, con las consecuentes catástrofes naturales. Para mantener el equilibrio entre el desarrollo tecnológico y el medio ambiente se acuñó, en 1987, el término "desarrollo sostenible". Dicho término fue definido como: forma de progreso que satisface las necesidades del presente, sin comprometer las necesidades de las futuras generaciones en todos los aspectos: social, humano y ambiental. A raíz de esto, se han llevado a cabo diversas convenciones con el reto de promover el desarrollo sostenible, siendo el protocolo de Kyoto, firmado en 1997, el tratado más relevante.

Para colaborar con el desarrollo sostenible, la industria del cemento está buscando soluciones dentro de su propio proceso de producción, puesto que en el ámbito de la construcción civil la industria cementera es la responsable de la mayor parte de las emisiones de $\mathrm{CO}_{2}$, así como de los niveles más elevados de consumo de energía y de materias primas no renovables. Se puede encontrar diversas alternativas para minimizar los problemas generados por la fabricación del cemento Pórtland. Una de las opciones planteadas es la utilización de combustibles alternativos que generen, en el proceso de producción del cemento Pórtland, menos emisiones contaminantes que los materiales actualmente empleados. Otra posibilidad consistiría en la mejora de la eficacia de los hornos utilizados en la producción del cemento Pórtland, pero para ello resultaría necesaria una gran inversión económica en el sector, hecho que hace inviable, en cierta medida, la puesta en marcha de esta alternativa de forma generalizada. Teniendo en cuenta las limitaciones que presentan las propuestas anteriormente mencionadas, actualmente se defiende como mejor alternativa para reducir el consumo de energía y la emisión de $\mathrm{CO}_{2}$, la utilización de adiciones minerales tanto en el proceso de fabricación del cemento Pórtland como en la producción de morteros y hormigones. De esta manera se reduciría, por una parte, el consumo de materias primas en la dosificación de crudos, y por otra parte, el consumo de clínker Pórtland y, todo ello lleva como consecuencia una reducción en las emisiones de $\mathrm{CO}_{2}$.

Las adiciones minerales puzolánicas son aquellos materiales, que si bien no presentan actividad cementicia por sí solos, al ser finamente divididos y mezclados con el agua reaccionan con el hidróxido de calcio a temperatura ambiente para formar compuestos con propiedades cementantes. 
Revista ALCONPAT, Volumen 2, Número 2, Mayo - Agosto 2012, Páginas 57 - 71

Estos materiales, compuestos mayoritariamente de sílice $\left(\mathrm{SiO}_{2}\right)$ y/o alúmina $\left(\mathrm{Al}_{2} \mathrm{O}_{3}\right)$, pueden ser residuos agrícolas, industriales o bien tratarse de productos sintetizados, como es el caso del metacaolín. Por todo ello, existe una amplia variedad de materiales que pueden ser utilizados como adiciones minerales puzolánicas, entre los cuales se puede destacar la ceniza volante $(\mathrm{CV})$, la ceniza de lodo de depuradora (CLD), el humo de sílice (HS), el residuo de catalizador de craqueo catalítico del petróleo (FCC), la ceniza de cáscara de arroz (CCA) (Ordóñez, 2007), el aluminosilicato cálcico vítreo (VCAS), o el metacaolín(MK), entre otros.

La CV es un residuo que se obtiene por precipitación electrostática o mecánica de partículas pulverulentas arrastradas por flujos gaseosos de hornos alimentados por carbón pulverizado en las centrales térmicas de generación de energía (Amahjour, 2000). El lodo de depuradora es un subproducto de las plantas de tratamiento de aguas residuales urbanas. Como resultado de la incineración de los lodos de depuradora, mediante la cual los compuestos orgánicos son descompuestos, se obtiene la ceniza de lodo de depuradora (CLD) con un volumen de sólo el 10\% del residuo original (Garcés, 2008 y Pérez-Carrión, 2008). El humo de sílice (HS), es un subproducto del proceso de obtención del silicio y de la aleación de ferrosilicio. En el proceso, se genera un humo que contiene micropartículas esféricas de dióxido de silicio en estado amorfo. El FCC es un material de desecho proveniente de las refinerías petróleo, concretamente de las plantas de craqueo catalítico en lecho fluido (Zornoza, 2007). Tiene una estructura zeolítica y está compuesto básicamente de aluminosilicatos (Garcés, 2011). El aluminosilicato cálcico amorfo (VCAS), es un residuo industrial proveniente del procesamiento de los residuos obtenidos en la fabricación de las fibras de vidrio. Se trata de un material vítreo, rico en óxidos de silicio, aluminio y calcio (Mitsuuchi, 2012).

La reactividad de las adiciones minerales puzolánicas debe ser evaluada en base a dos parámetros principales: la cantidad de hidróxido de calcio combinado con la puzolana y la velocidad a la que se consume dicha cal. Estos parámetros están directamente relacionados con la naturaleza y calidad de la puzolana.

Quizás uno de los aspectos que genera mayores dudas en relación a la utilización de estas puzolanas, es que el desarrollo de su potencial actividad puzolánica implica el consumo, en mayor o menor medida, del $\mathrm{Ca}(\mathrm{OH})_{2}$ generado en la hidratación de la fases anhidras del cemento. Y por lo tanto, el consumo de la reserva alcalina, el $\mathrm{Ca}(\mathrm{OH})_{2}$ o portlandita, presente en las paredes de los poros y en la disolución intersticial contenida en el interior de la pasta cementicia. Es esta reserva alcalina la que, en principio, garantiza la estabilidad de la capa pasivante que preserva al acero de refuerzo frente a la corrosión en las estructuras de hormigón armado.

El objetivo de este artículo es presentar un resumen de trabajos de investigación donde se ha evaluado el nivel de corrosión que presentan armaduras embebidas en morteros y hormigones donde se ha sustituido parcialmente el cemento por diferentes residuos caracterizados por su actividad puzolánica. Asimismo, se presentan características resistentes de algunos de los conglomerantes en base cemento Portland fabricados con sustitución del cemento por estos residuos con actividad puzolánica. La información se ha obtenido a partir de Tesis Doctorales (Amahjour, 2000; Ordoñez, 2007; Zornoza, 2007; Pérez-Carrión, 2008) y diferentes publicaciones, todos ellas desarrolladas en el ámbito del grupo de excelencia de la Generalitat Valenciana: Unidad de investigación y Ciencia del Hormigón (UNICH), formado por profesores de los Departamentos de Ingeniería de la Construcción de la Universidad de Alicante y la Universitat Politècnica de València en España. Dicho grupo trabaja en la línea de la sostenibilidad en el ámbito de la construcción. 
Revista ALCONPAT, Volumen 2, Número 2, Mayo - Agosto 2012, Páginas 57 - 71

\section{EXPERIMENTAL}

Los resultados que se presentan a continuación se han obtenido utilizando los siguientes materiales: Portland tipo CEM I 52.5 R, arena silícea normalizada cumpliendo la norma europea UNE-EN 196-1, árido calizo, FCC (suministrado por BP España), CV (suministrada por la central térmica de Andorra-España) y CLD (suministrada por la planta de Pinedo-Valencia de tratamiento de aguas residuales urbanas). La Tabla 1 muestra la composición química de los residuos utilizados.

Tabla 1. Composición química de los residuos en porcentajes.

\begin{tabular}{|l|c|c|c|c|c|c|c|c|c|}
\cline { 2 - 10 } \multicolumn{1}{c|}{} & $\mathrm{SiO}_{2}$ & $\mathrm{Al}_{2} \mathrm{O}_{3}$ & $\mathrm{Fe}_{2} \mathrm{O}_{3}$ & $\mathbf{C a O}$ & $\mathrm{K}_{2} \mathrm{O}$ & $\mathrm{Na}_{2} \mathrm{O}$ & $\mathrm{MgO}$ & $\mathrm{SO}_{3}$ & $\mathrm{PF}^{*}$ \\
\hline $\mathrm{CV}$ & 40.94 & 24.65 & 13.59 & 9.83 & 1,40 & 0.34 & 1.59 & 1.60 & 0.49 \\
\hline FCC & 46.04 & 47.47 & 0.58 & 0.11 & - & - & 0.17 & 0.02 & 2.44 \\
\hline CLD & 17.9 & 17.0 & 16.6 & 26.7 & - & - & 1.99 & 11.7 & 2.19 \\
\hline
\end{tabular}

*Pérdida al fuego a $950^{\circ} \mathrm{C}, 1$ hora.

\section{RESULTADOS Y DISCUSIÓN}

En primer lugar presentaremos algunos de los resultados obtenidos mediante la incorporación del CLD a morteros, en lo que respecta a la corrosión de armaduras (Garcés, 2006). La figura 1 muestra la evolución de la densidad de corriente de corrosión correspondiente a las barras de acero de refuerzo embebidas en los morteros fabricados con cemento portland y diferentes proporciones de ceniza de lodo de depuradora (CLD): 0, 10, 20, 30 y 60\% (porcentajes de sustitución en relación al peso de cemento) y siendo el agua/material cementante $(\mathrm{a} / \mathrm{mc}) 0.7$. Tras el curado en cámara húmeda durante 28 días, estos morteros permanecieron durante todo el tiempo de experimentación parcialmente sumergidos en agua de mar. La franja rojiza horizontal dibujada entre los valores de 0.1 y $0.2 \mu \mathrm{A} / \mathrm{cm}^{2}$, es el umbral de corrosión. Por debajo se considera la corrosión despreciable, y por encima, la velocidad de corrosión es lo suficientemente grande como para poder afectar a la vida útil de la estructura. Es interesante destacar que en estas probetas sumergidas en agua de mar se observa que los morteros con un 10 y $20 \%$ de CLD tienen un comportamiento similar al del mortero de control, situándose por debajo de la zona de corrosión. Únicamente para aquel mortero con una sustitución parcial del cemento por CLD del 60\%, los niveles de corrosión registrados son altos. Hay que tener en cuenta que para esta sustitución tan alta, la trabajabilidad del mortero era muy reducida y el nivel de porosidad muy alto en comparación con el mortero control y con los morteros con sustituciones del 10 y $20 \%$. 


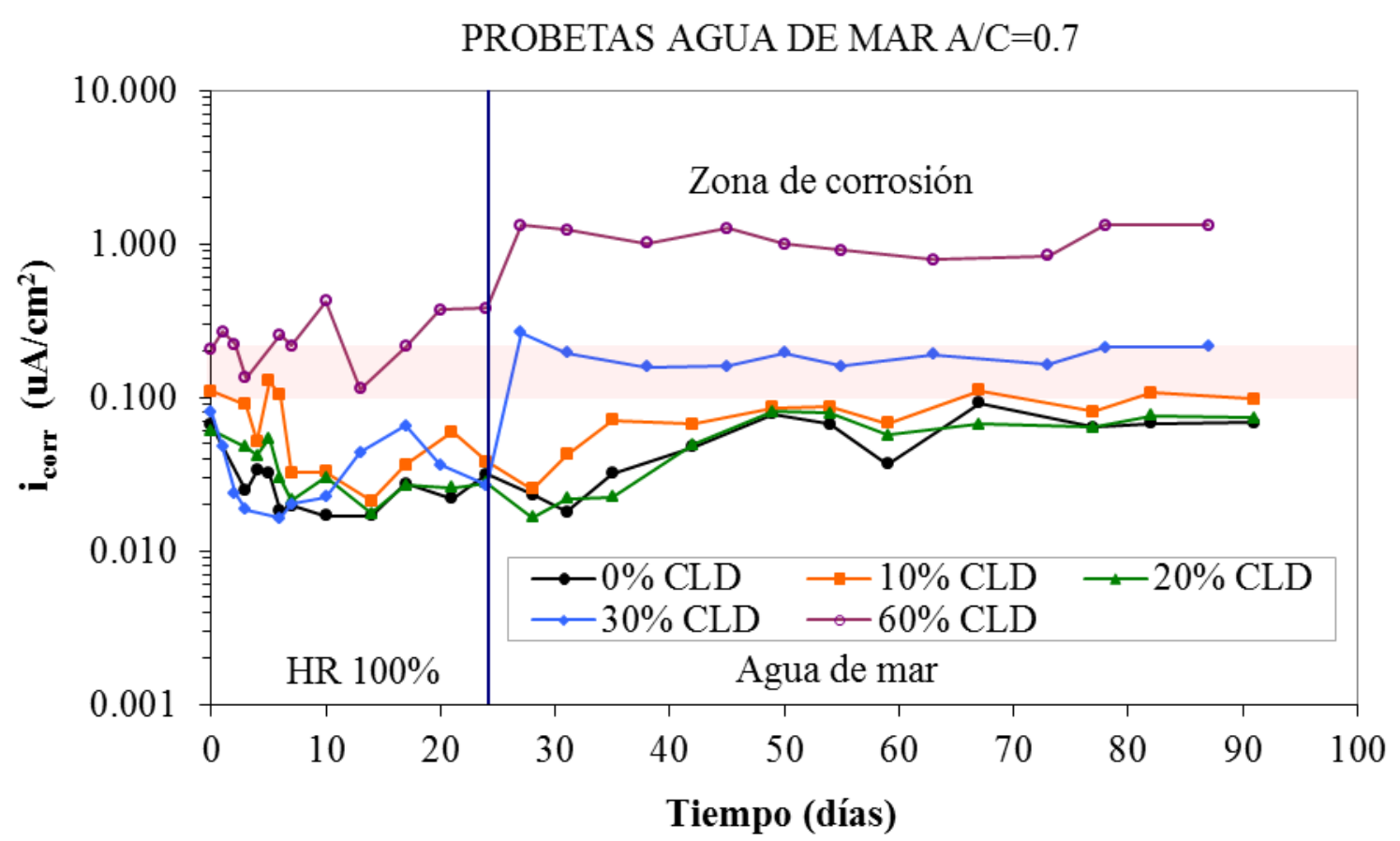

Figura 1. Evolución de la velocidad de corrosión en probetas confeccionadas con 0, 10, 20, 30 y $60 \%$ de sustitución de cemento Pórtland por CLD, relación a/mc=0.7 y sumergidas en agua de mar.

En el siguiente caso se utilizó CV y algunos productos que fueron obtenidos como resultado de la aplicación de diversos pretratamientos (Garcés, 2010). La figura 2 muestra la evolución de la densidad de corriente y el potencial de corrosión correspondiente a las barras de acero de refuerzo embebidas en los morteros fabricados con cemento portland, con relaciones a/mc: 0.5 y 0.7 (patrones) y con sustitución parcial (30\%) del cemento por ceniza volante (CV) original, CV sometida a molienda durante 60 minutos, la fracción magnética TM y no magnética TNM de la misma ceniza volante, obtenidas al someter a la acción de un campo magnético la CV. Tras el curado, estos morteros permanecieron durante todo el tiempo de experimentación parcialmente sumergidos en agua de mar. Como se puede observar en la figura 2, el comportamiento de los aceros en cada una de las matrices cementicias estudiadas es significativamente diferente. Cabe destacar, en primer lugar, que el nivel de corrosión que presentan los aceros embebidos en el mortero con sustitución del $30 \%$ del cemento por ceniza volante original (T0) se sitúan entre lo registrado en el patrón con a/mc $0.5 \mathrm{y} \mathrm{a} / \mathrm{mc} 0.7$, habiéndose producido en todos ellos la despasivación y por tanto el desarrollo de la pila de corrosión. Por lo tanto, una capa de recubrimiento porosa, por exceso de agua en el amasado, puede tener una influencia más negativa que la sustitución parcial de cemento por CV. Resulta muy interesante, por otra parte, el que en las condiciones agresivas planteadas en este trabajo, el mortero con CV sometida a molienda y con la fracción no magnética de la $\mathrm{CV}$, mantenga el refuerzo pasivado durante toda la experiencia. 
Revista ALCONPAT, Volumen 2, Número 2, Mayo - Agosto 2012, Páginas 57 - 71
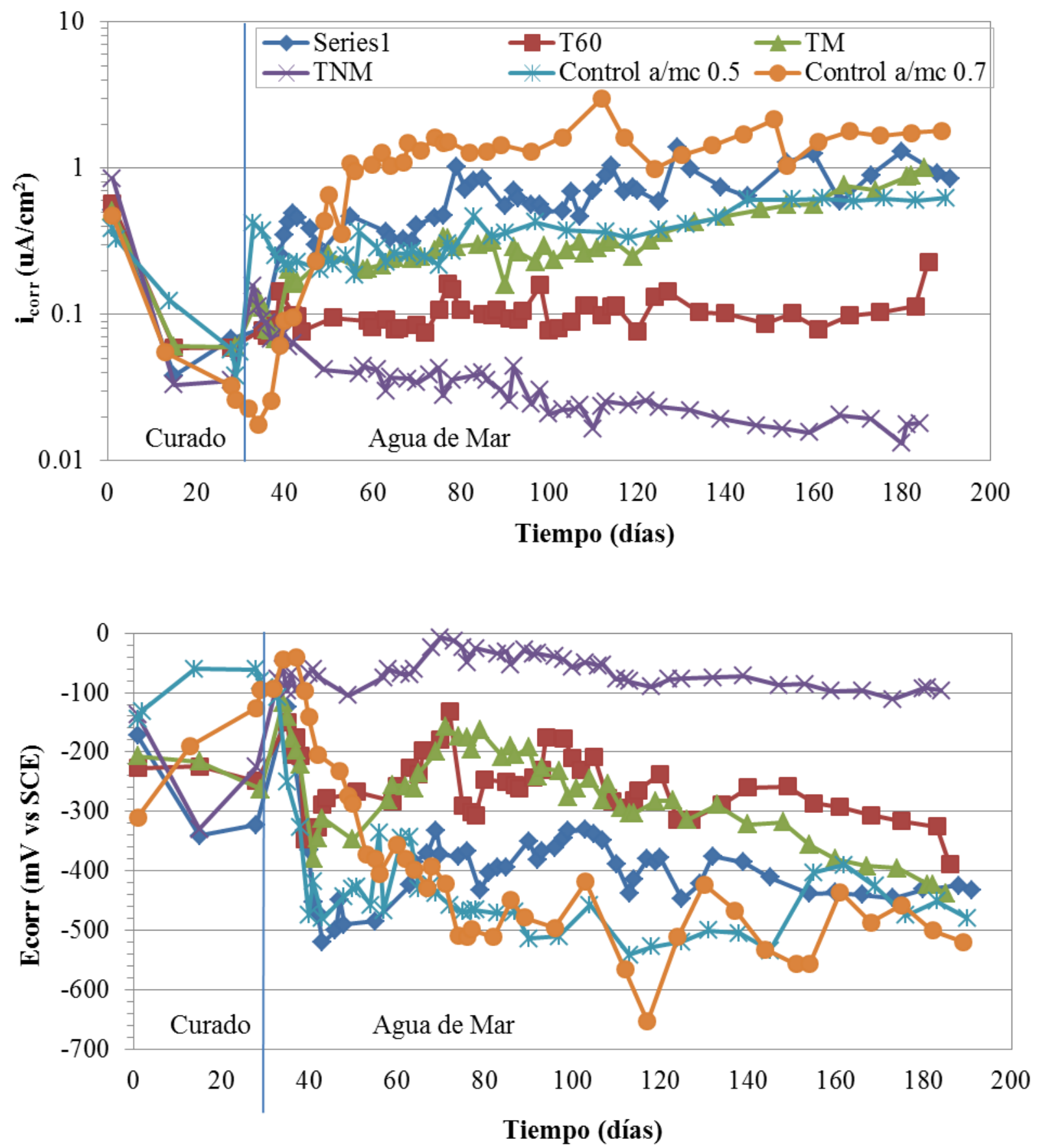

Figura 2. Evolución de la densidad de corriente y potencial de corrosión con el tiempo para probetas de mortero curadas durante 28 días, humedad relativa del $100 \%$ y sometida a inmersión parcial en agua de mar. En todos los casos sustitución del 30\% de cemento por CV. T0: Mortero con CV original, T60: Mortero con CV sometida a molienda 60 minutos, TM: Mortero con fracción magnética de CV, TNM: Mortero con fracción no magnética de CV, comparados con patrones de a/mc 0.5 y 0.7 . 
Revista ALCONPAT, Volumen 2, Número 2, Mayo - Agosto 2012, Páginas 57 - 71

Así pues, la utilización de este tipo de materiales no está reñida con el cumplimiento de los requisitos de durabilidad en términos de corrosión de armaduras. No obstante, lo que sí exige, como en casi cualquier otro material, es la utilización del conocimiento previo que se tenga para seleccionar adecuadamente tanto el ámbito de aplicación, como los límites que se deben emplear en el uso de los mismos.

Profundizando un poco más, también podemos tener un importante aliado en la utilización de estos subproductos a la hora de mejorar algunas propiedades que tienen gran impacto en la durabilidad, en concreto, en lo que hace referencia a la mejora de la microestructura. Por ello es importante destacar el efecto que sobre la densificación de la matriz cementante puede tener lugar en el desarrollo de los productos cementantes derivados de la actividad puzolánica de algunos residuos. Así, en la figura 3 se observan los valores de las intrusiones de mercurio acumuladas de los morteros (ensayos de porosimetría de intrusión de mercurio), en función de su relación a/mc y de la presencia de FCC. En ella se aprecia cómo la intrusión acumulada final (el valor obtenido para el diámetro más pequeño) sigue un orden creciente según aumenta la relación a/mc. Esto es lógico, ya que la porosidad de los morteros aumenta según aumenta la relación a/mc.

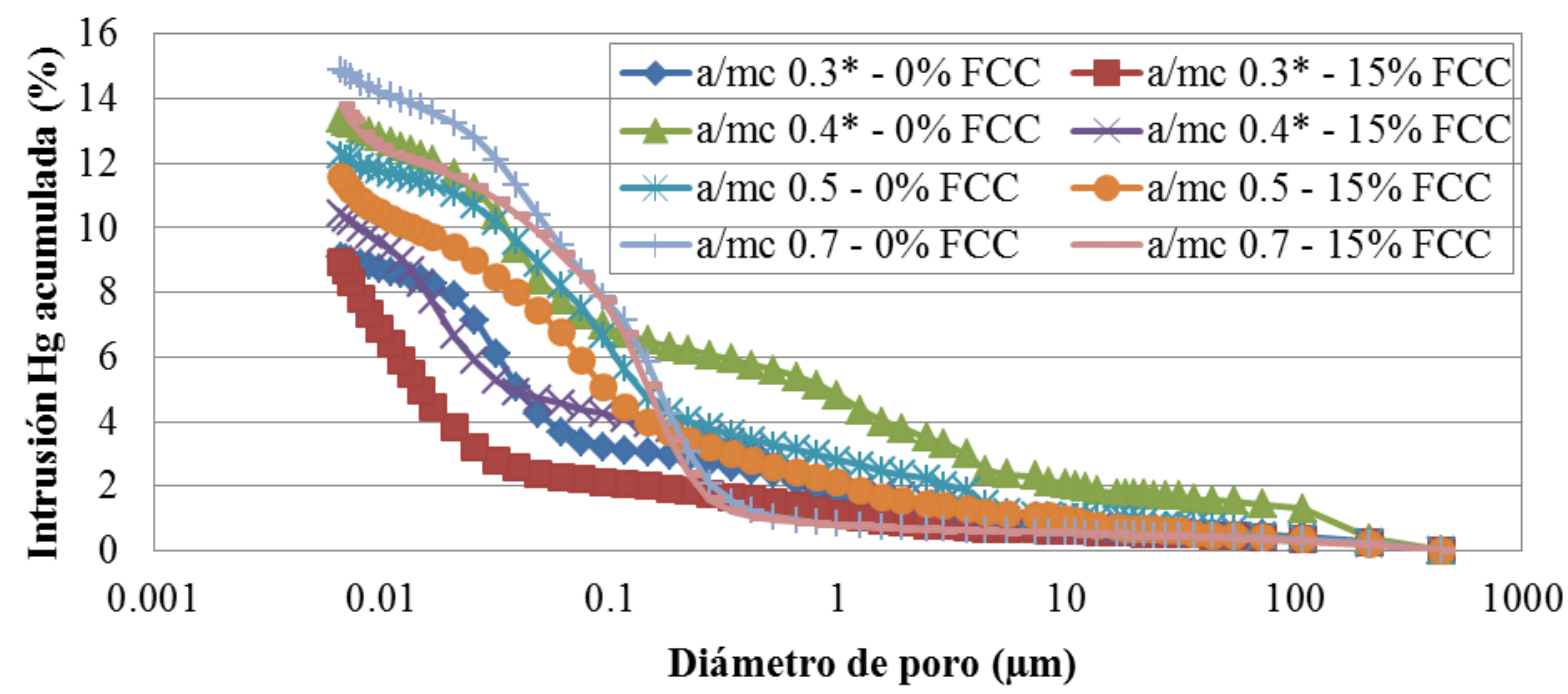

Figura 3. Distribución de tamaños de poros en los morteros de cemento/FCC en función de la relación a/mc.

También se observa en dicha gráfica que la sustitución de cemento por FCC produce una reducción de la porosidad total (ver nuevamente el valor de la intrusión acumulada final). Esto es debido a la densificación que se produce en la probeta por la formación de productos cementantes, a consecuencia de la reacción puzolánica entre el FCC y la portlandita liberada por la hidratación del cemento. Por otra parte, si se considera como porosidad capilar la comprendida en el intervalo de $50 \mathrm{~nm}$ a 3 micras, se puede observar (Figura 4, Zornoza 2009b) que existe una clara dependencia de la porosidad capilar con la relación a/mc y además el FCC produce un descenso sistemático de dicha porosidad capilar, la cual afecta notablemente a los procesos de transporte a través del mortero. 
Revista ALCONPAT, Volumen 2, Número 2, Mayo - Agosto 2012, Páginas 57 - 71

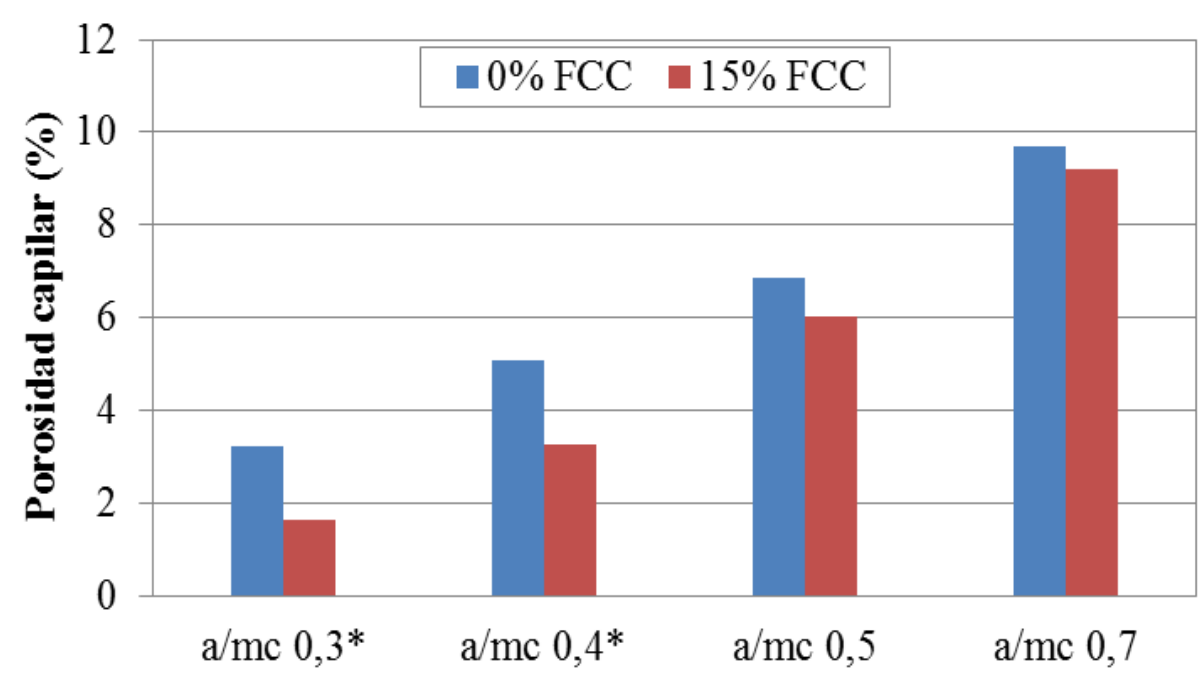

Figura 4. Distribución de tamaños de poros capilares (responsables de los fenómenos de transporte) en los morteros de cemento/FCC en función de la relación a/mc.

A la vista de estos resultados, cabe cuestionarse sobre cuál es el impacto real de estos cambios microestructurales en los fenómenos de transporte, como la difusión de $\mathrm{CO}_{2}$. Son muchos los modelos de predicción de vida de servicio de estructuras en función de la velocidad de avance de la carbonatación en elementos en servicio. Estos modelos pueden ser más o menos completos e intentan abarcar todos los factores que influyen en la velocidad de carbonatación, como pueden ser: la humedad ambiental, el contenido de portlandita de los hormigones, el grado de hidratación del cemento, etc.

En los resultados que se presentan a continuación, se pretende comparar cómo influyen la sustitución de cemento por residuo de FCC y la relación a/mc en la velocidad de carbonatación de los morteros. Para ello se presentan resultados que se han ajustado mediante el modelo clásico que relaciona la profundidad de carbonatación con la raíz cuadrada del tiempo mediante una constante, que denominamos velocidad de carbonatación, y cuya ecuación responde a la siguiente expresión:

$$
x=V_{\mathrm{CO} 2} \sqrt{t}
$$

Con los resultados experimentales obtenidos (figura 5) se calculó el coeficiente $\mathrm{V}_{\mathrm{CO} 2}$ que sirvió para comparar la resistencia que ofrecen los distintos morteros ante el avance del frente de carbonatación.

En la figura 5 se puede observar que a medida que se aumenta la relación a/mc, la velocidad de carbonatación crece considerablemente (Zornoza, 2009a). Este fenómeno es esperable debido a que la porosidad de los morteros aumenta al incrementar la relación a/mc, y por lo tanto el $\mathrm{CO}_{2}$ encuentra el camino mucho más despejado para penetrar hacia el interior del mortero. En las relaciones a/mc bajas ( 0,3 y 0,4 con plastificante) la presencia del FCC no ofrece diferencias apreciables con respecto a los morteros sin residuo de FCC. 
Revista ALCONPAT, Volumen 2, Número 2, Mayo - Agosto 2012, Páginas 57 - 71

En cambio, para las relaciones a/mc 0,5 y 0,7, el residuo de FCC empeora la resistencia del mortero frente a la carbonatación. Parece ser que la reducción de la reserva alcalina debido al consumo de la misma por el FCC es bastante importante, y ello conlleva una menor cantidad de sustancias carbonatables que frenen el avance de la carbonatación aumentando las constantes de carbonatación (figura 6).

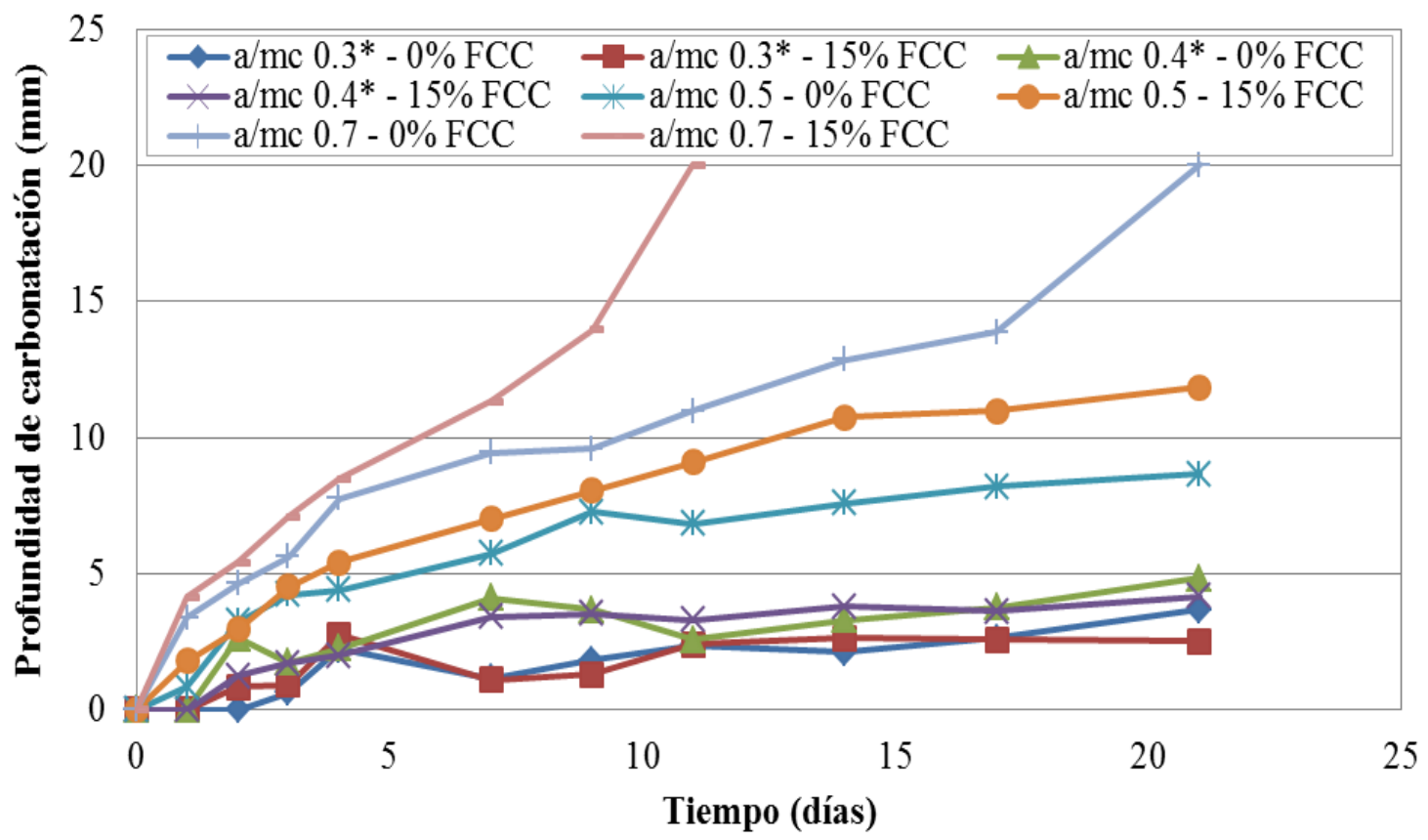

Figura 5. Evolución del perfil de carbonatación de morteros durante el ensayo de carbonatación acelerada.

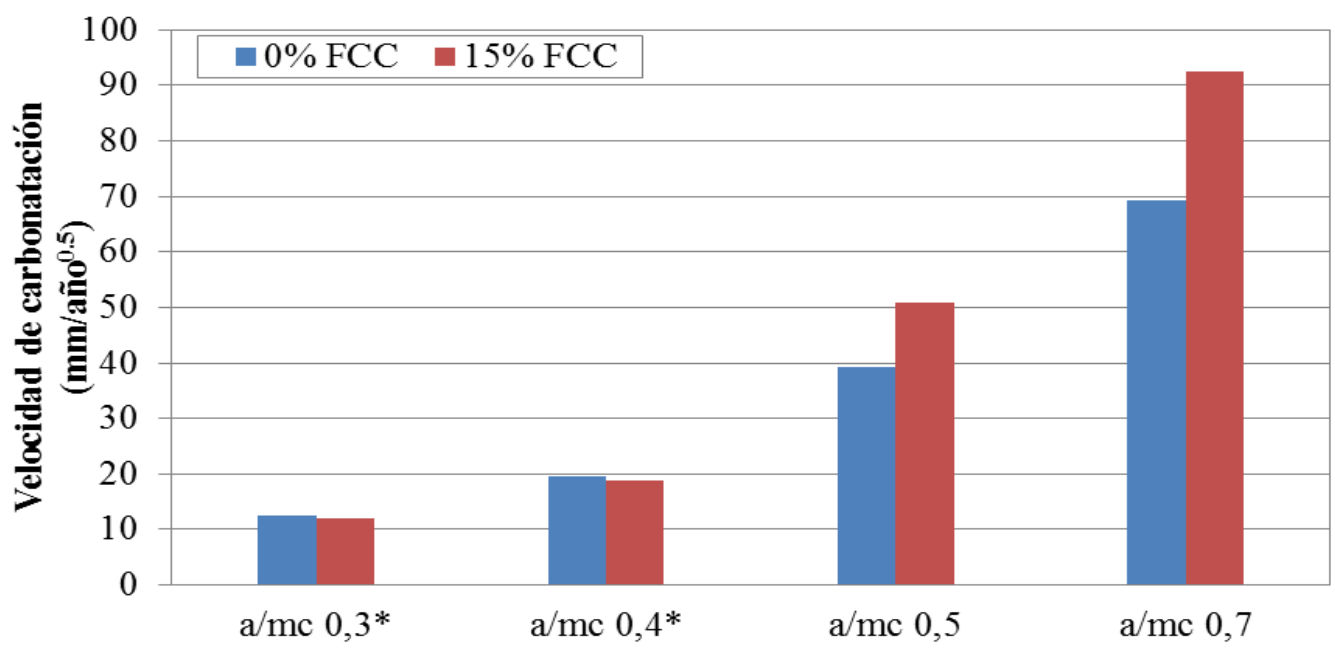

Figura 6. Velocidad de carbonatación de los morteros en el ensayo de carbonatación acelerada.

A su vez, no sólo las propiedades de transporte de los productos elaborados son modificadas, también lo son, y es de sobra conocido, el impacto en las propiedades mecánicas. Eso sí, el resultado dependerá de cada material en concreto, aunque no nos debe preocupar en exceso ya que existe sobrada información sobre la influencia de cada puzolana en las propiedades mecánicas. Esto es así ya que tradicionalmente, es la principal propiedad por la que pregunta un ingeniero 
Revista ALCONPAT, Volumen 2, Número 2, Mayo - Agosto 2012, Páginas 57 - 71

civil, y efectivamente, es la primera función que debe ser asumida por el material: resistir un esfuerzo. En la figura 7 se presentan los resultados de resistencia a compresión de estos mismos morteros (Zornoza, 2008b y 2009a). Se puede comentar que a medida que aumenta la relación a/mc se produce un descenso de la resistencia a compresión, debido al aumento de la porosidad, y que además la incorporación de FCC produce un significativo incremento en este parámetro como consecuencia de la mejora de la microestructura del mortero producida por la formación de productos cementantes adicionales.

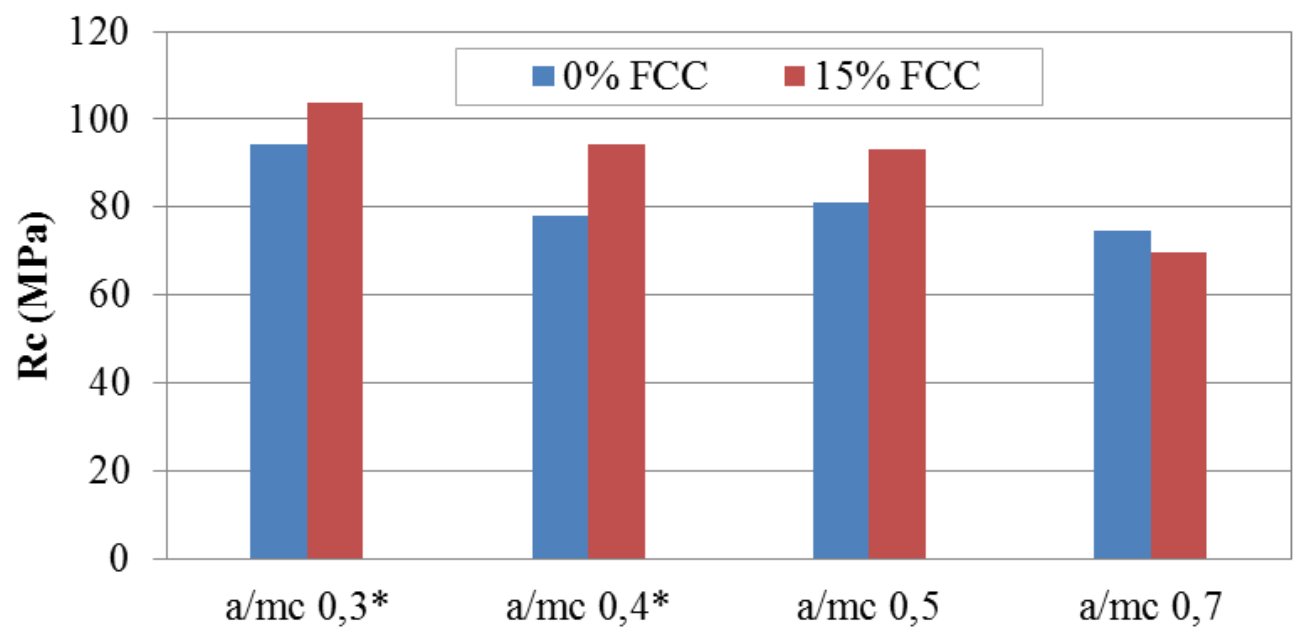

Figura 7. Datos de resistencia a compresión de los morteros patrón y con sustitución de un 15\% de cemento por FCC.

En algunos casos, además, se pueden encontrar agradables sorpresas, ya que existen materiales disponibles, como el residuo de FCC, que no sólo no reducen alguna de las propiedades relacionadas con la durabilidad, sino que además son capaces de mejorarlas significativamente. En las figuras 8 y 9 se presentan los resultados obtenidos en los estudios de corrosión tanto para los morteros sin FCC como para los que incorporan un 15\% de FCC, para diferentes contenidos de cloruros en la matriz cementante (Zornoza, 2009c). Lo primero que se aprecia de forma muy evidente es la ordenación de los valores de velocidad de corrosión y potencial de corrosión en función de la concentración de cloruros. A medida que aumenta dicha concentración se produce un desplazamiento del potencial hacia valores más negativos y un aumento de la velocidad de corrosión. Además se pueden extraer interesantes observaciones. Si se observan los valores en torno a los que se estabilizan las velocidades de corrosión, para el mortero control se podría establecer el umbral de cloruros entre el 1 y el $2 \%$ de cloruros respecto del peso de cemento, mientras que para el mortero con FCC dicho umbral se situaría entre el 2 y el 5\%, lo cual supone una importante mejora en el nivel de cloruros tolerable. 
Revista ALCONPAT, Volumen 2, Número 2, Mayo - Agosto 2012, Páginas 57 - 71
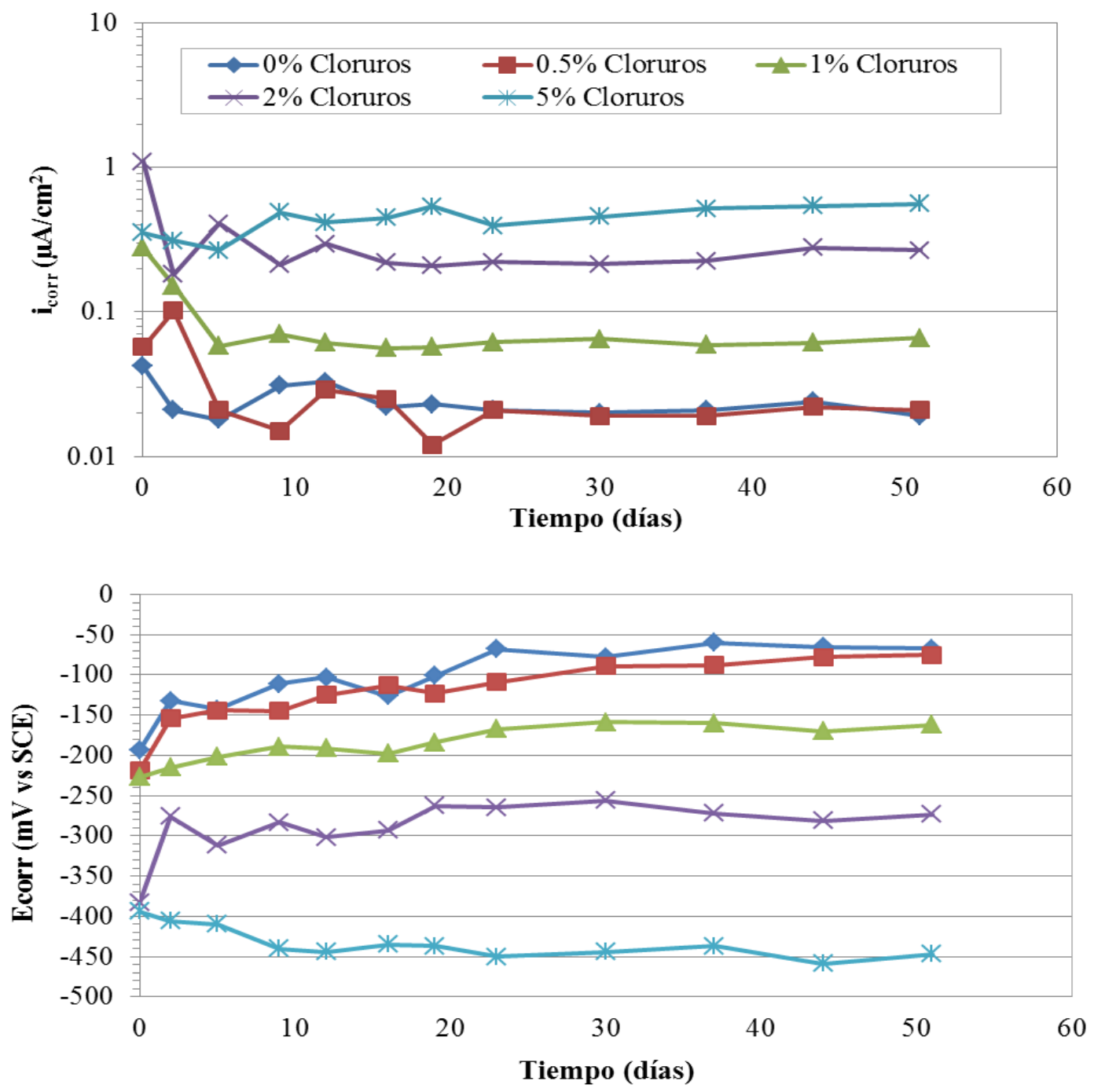

Figura 8. Niveles de corrosión del acero registrados en morteros patrón (100\% cemento) para diferentes porcentajes de cloruros añadidos al agua de amasado.

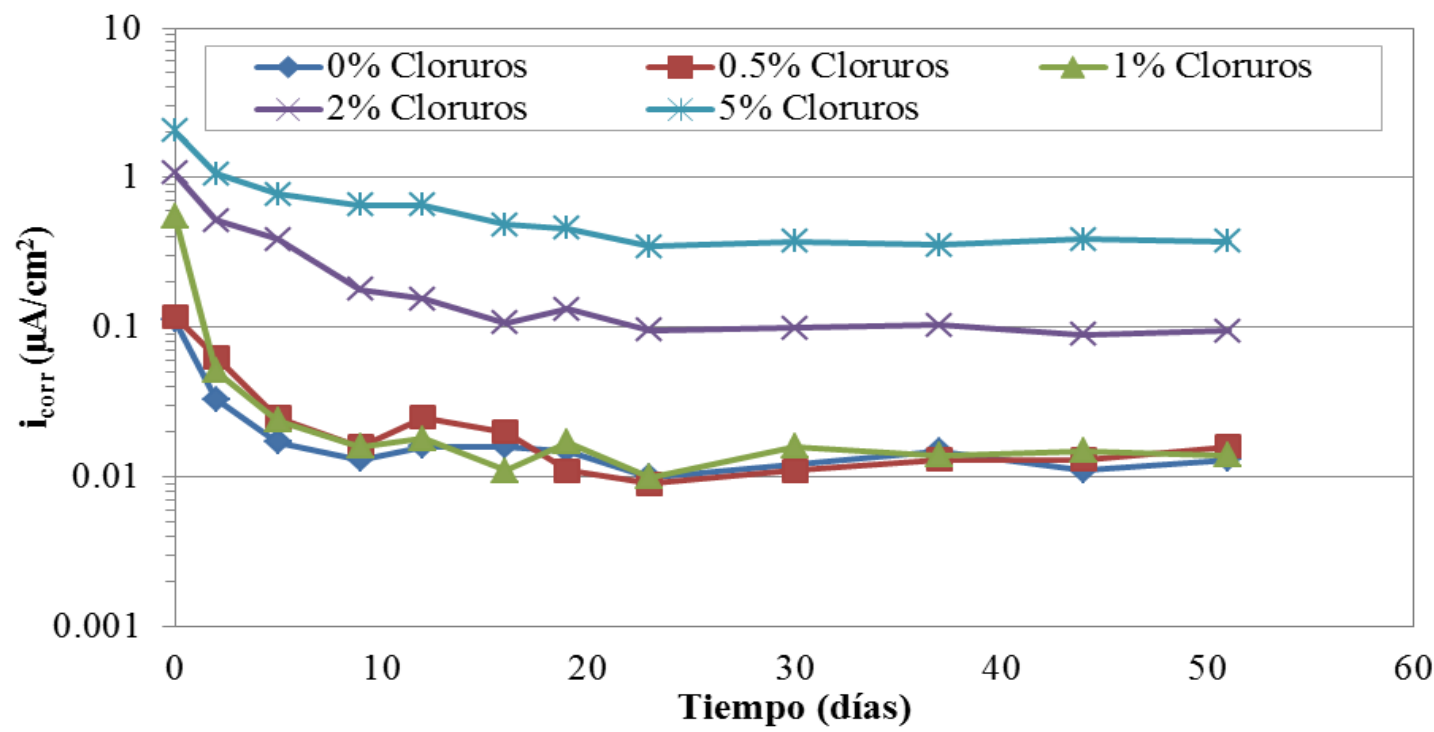

¿Es compatible la durabilidad con la sostenibilidad en la industria de la construcción? 
Revista ALCONPAT, Volumen 2, Número 2, Mayo - Agosto 2012, Páginas 57 - 71

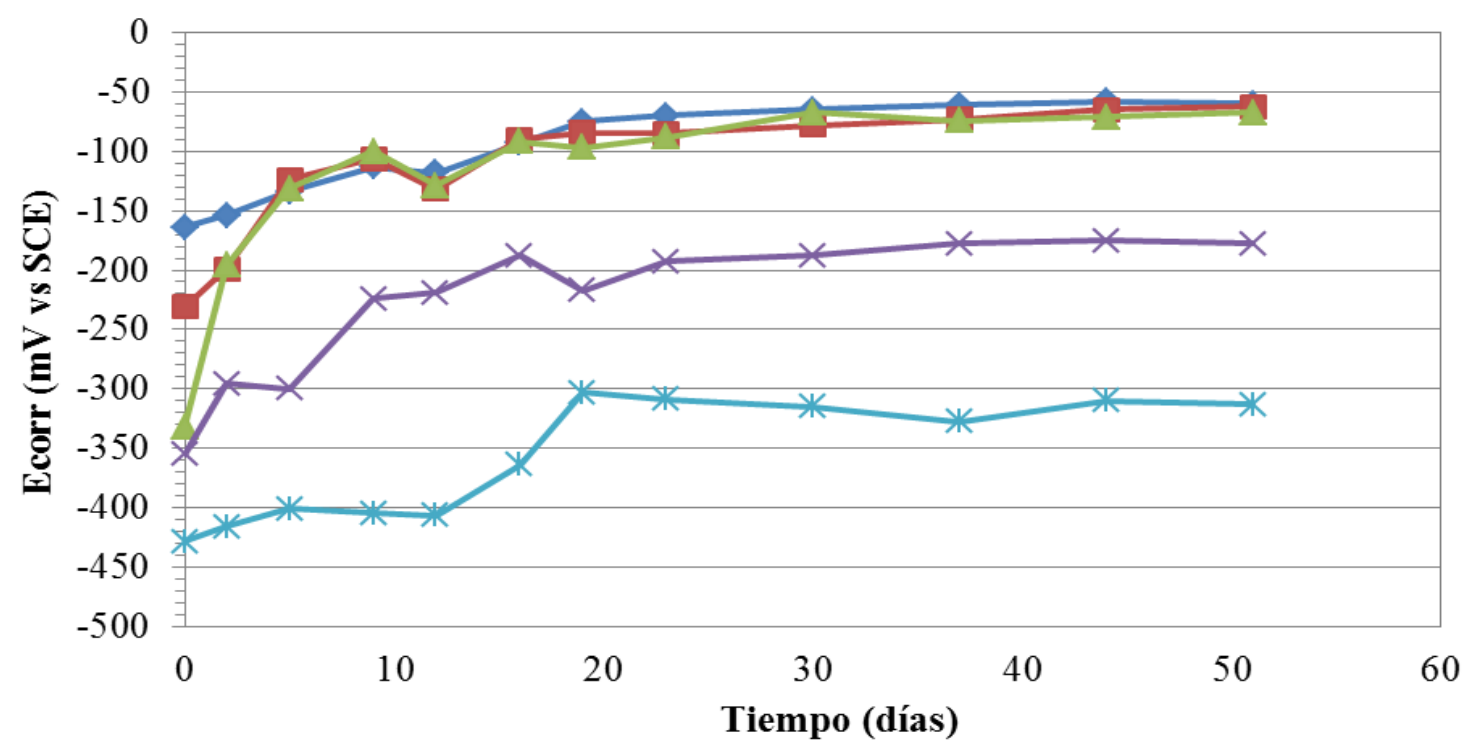

Figura 9. Niveles de corrosión del acero registrados en morteros con sustitución de un 15 del cemento por FCC para diferentes porcentajes de cloruros añadidos al agua de amasado.

Por último, una de las posibilidades que tiene más interés potencial es el diseño de mezclas ternarias de cemento con dos residuos que presenten propiedades complementarias. Tal es el caso de la CV y el FCC. A continuación se presentan resultados obtenidos del estudio de resistencia al ingreso de cloruros y corrosión de armaduras en morteros ternarios de cemento, FCC y ceniza volante. La presencia de ceniza volante en morteros y hormigones mejora la trabajabilidad de los morteros por lo que se puede compensar la alta demanda de agua que se observa que presenta el FCC.

Además mediante este tipo de mezclas cementantes se pueden conseguir porcentajes de sustitución de cemento muy elevados, lo que conlleva beneficios medioambientales, a la par que se consigue mejorar las propiedades mecánicas de los productos elaborados y mejorar la microestructura de la matriz cementante. Por otro lado, como ya se estudió anteriormente, usar sustituciones tan elevadas de cemento puede ocasionar una reducción o agotamiento de la reserva alcalina, lo que pondría en peligro la correcta pasivación de las armaduras. Se han comparado morteros con diferentes sustituciones de cemento: $30 \%$ para la $\mathrm{CV}, 15 \%$ para el FCC, y una combinación de $30 \%$ de CV más $15 \%$ de FCC para el mortero diseñado con mezcla de puzolanas. La figura 10 muestra la evolución de la velocidad de corrosión y el potencial de corrosión de los aceros embebidos en morteros de cemento-FCC-CV durante el ataque por cloruros externos al que fueron sometidas las muestras en la que la relación a/mc fue de 0,5 (Zornoza, 2008a y 2009d). En la primera fase se aprecia que inicialmente la velocidad de corrosión de los aceros es elevada debido al proceso de pasivación de los aceros. Progresivamente los valores se reducen hasta situarse por debajo del umbral de corrosión activa.

Durante el ataque por cloruros, las probetas se sumergieron parcialmente en una disolución de $\mathrm{NaCl}$ 0,5 M. En este caso se observa que los perfiles de la velocidad de corrosión presentan los dientes de sierra típicos de un ataque por cloruros que originan picaduras en la superficie del acero. En general, los morteros con sustitución de cemento por FCC y/o CV ofrecen valores de velocidad de corrosión inferiores a los del mortero Control pero no se puede discriminar entre ellos ninguno que destaque con respecto a los demás.

Los valores de velocidad de corrosión de los morteros con FCC y/o CV permanecen siempre por debajo del umbral de corrosión activa. La presencia de FCC en los morteros mejora sensiblemente la capacidad de fijación de cloruros de la matriz cementante mientras que la incorporación de un 
Revista ALCONPAT, Volumen 2, Número 2, Mayo - Agosto 2012, Páginas 57 - 71

$30 \%$ de CV lo hace ligeramente, debido a que el aporte de aluminatos por el FCC es muy superior al aporte de la CV. En otro estudio se comprobó que la mezcla ternaria de cemento Portland-FCCceniza volante consigue reducir apreciablemente el coeficiente de difusión de estado estacionario de los morteros (a pesar de contener mucho menos cemento), aunque esta mejora no es tan notable como en el caso de incorporar únicamente FCC.
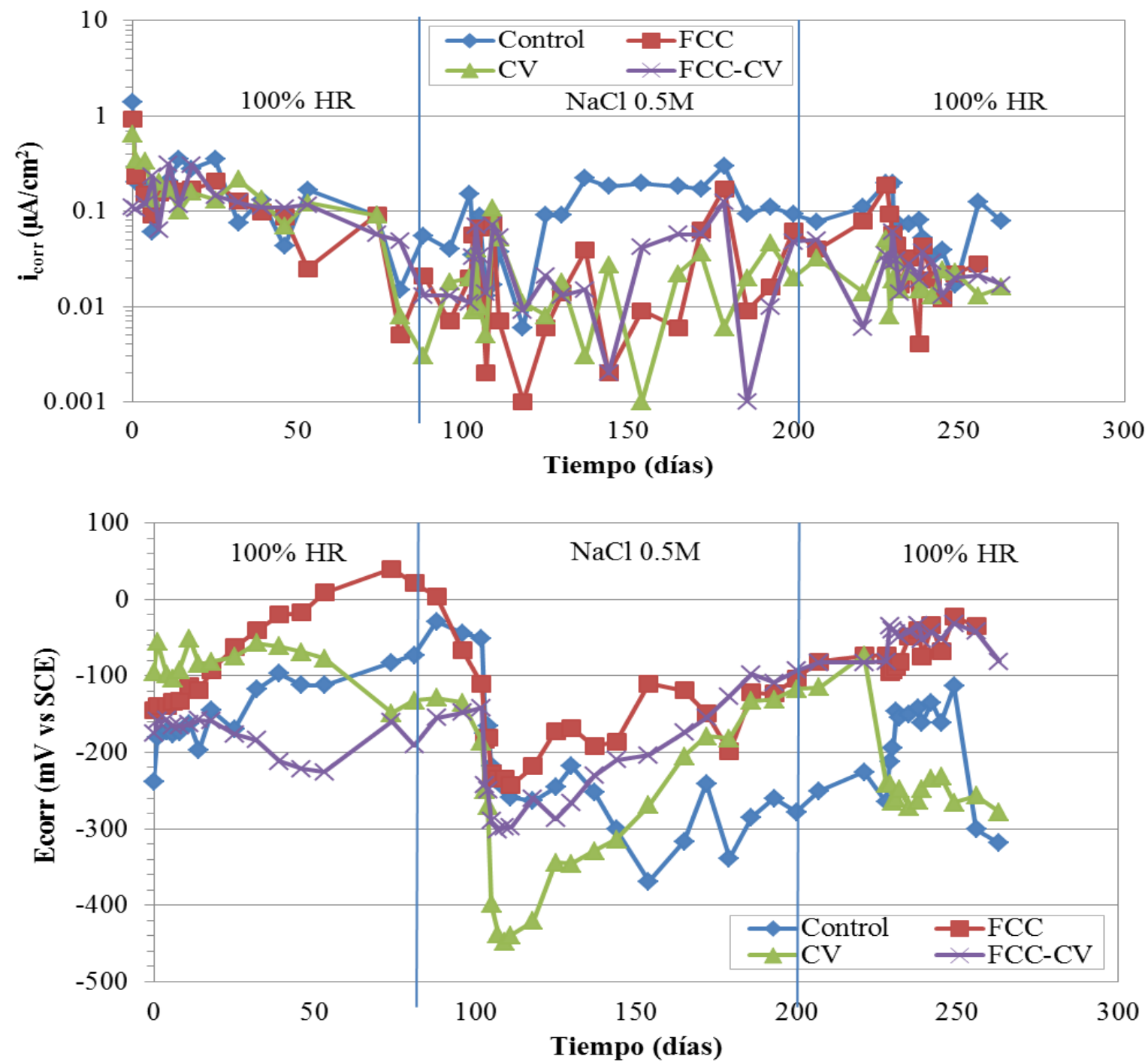

Figura 10. Evolución de la velocidad de corrosión y el potencial de corrosión de los aceros embebidos en morteros de cemento-FCC-CV durante el ataque por cloruros externos. Relación $\mathrm{a} / \mathrm{mc}$ de 0,5 .

Otra de las mezclas ternarias que se han estudiado son las resultantes del sistema cemento Portland-CLD-CCA. En la figura 11 se presentan los valores obtenidos de resistencia a compresión en probetas de mortero con las siguientes formulaciones: 1) Patrón, 2) adición 10\% CCA, 3) sustitución del 30\% de cemento (20\%CLD+10\% CCA) y 4) 30\% (20\%CLD+10\%CCA) + adición 10\% CCA. Hay que destacar en primer lugar que todos los valores de resistencia característica a 28 días se encuentran por encima de la clase resistente del cemento. En segundo destaca que en el caso 4) el nivel de consumo de residuo es muy importante y sin embargo sigue superando la clase resistente del cemento utilizado (32.5 MPa). Por último, y como era de esperar 
Revista ALCONPAT, Volumen 2, Número 2, Mayo - Agosto 2012, Páginas 57 - 71

las resistencias disminuyen en todos los casos a medida que aumentan las relaciones a/mc consideradas.

Probetas de MORTERO

Resistencia a compresión - Valor absoluto medio 28 días

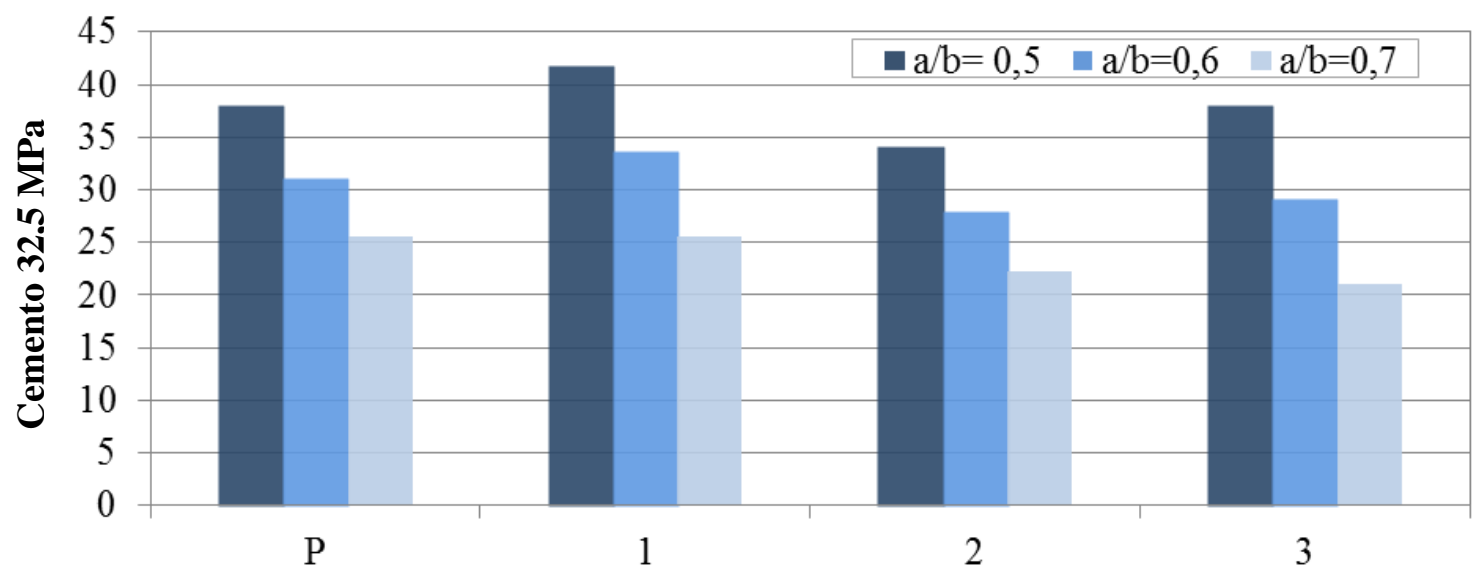

Figura 11. Resistencia a compresión en probetas de mortero CLD-CCA curadas durante 28 días con distintas relaciones agua/material cementante. 1) Patrón, 2) adición 10\% CCA, 3) sustitución del $30 \%$ de cemento (20\%CLD+10\% CCA) y 4) 30\% (20\%CLD+10\%CCA) + adición 10\% CCA.

De los resultados presentados en este trabajo se deriva claramente que la utilización de los denominados "ecoconglomerantes" basados en la sustitución de parte del cemento por residuos con actividad puzolánica no implica necesariamente una reducción de las características resistentes. Por otra parte, la adecuada combinación de cemento y puzolana junto con la pertinente selección del ámbito de aplicación de los mismos, no conlleva un empeoramiento de la durabilidad de los elementos fabricados con estos "ecoconglomerantes" en términos de corrosión de armaduras.

\section{CONCLUSIONES}

1. La utilización de residuos industriales o agrícolas con actividad puzolánica en las cantidades adecuadas pueden mantener o mejorar las propiedades mecánicas de los conglomerantes.

2. Se ha podido observar una disminución en la porosidad capilar de las matrices cementantes que incorporan residuos industriales o agrícolas, lo cual puede potencialmente reducir los fenómenos de transporte de agentes agresivos como el $\mathrm{CO}_{2}$ o los iones cloruro.

3. La adecuada combinación de residuos industriales o agrícolas sustituyendo parte del cemento puede preservar de forma eficaz la capa pasivante de la superficie de los aceros, por lo que garantiza su durabilidad en ambientes no agresivos.

4. La selección de ciertos residuos industriales o agrícolas permite obtener ventajas tecnológicas mejorando algunas propiedades relacionadas con la durabilidad como puede ser la capacidad de fijación de cloruros de la matriz cementante.

5. Es posible fabricar materiales conglomerantes compatibles con un desarrollo sostenible en el ámbito de la construcción. 
Revista ALCONPAT, Volumen 2, Número 2, Mayo - Agosto 2012, Páginas 57 - 71

\section{REFERENCIAS}

Amahjour, F. (2000) Tesis doctoral. Estudio de la reactividad de las puzolanas artificiales: cenizas volantes y humo de sílice y su reutilización en ingeniería civil. Universidad Politécnica de Valencia, Valencia.

Garcés, P., Perez Carrión, M., Alcocer, E. Ga ., Payá, J., Andión, L. G. (2006) Efecto de la adición de ceniza de lodo de depuradora (CLD) en las propiedades mecánicas y niveles de corrosión de armaduras embebidas en morteros de cemento Portland. Materiales de Construcción, Vol. 56, No. 282, pp 31-43.

Garcés, P., Perez Carrión, M., Alcocer, E. Ga., Payá, J. (2008) Mechanical and physical properties of cement blended with sewage sludge ashes. Waste management, Vol. 28, pp 2495-2502.

Garcés, P., Andión, L. Ga , Zornoza, E., Bonilla, M., Payá, J. (2010) The effect of processed fly ashes on the durability and the corrosion of steel rebars embedded in cement-modified fly ash mortars. Cement and concrete composites, Vol. 32, pp. 201-210.

Garcés, P., Glasser, F. P., Brew, D. R. M., Zornoza, E., Payá J. (2011) Pozzolanic Activity of a Spent Fluid Catalytic Cracking Catalyst Residue. Advances in Cement Research, Vol. 23, No. 1, pp. 1-7

Mitsuuchi, M. (2012) Tesis doctoral. Producción y caracterización de materiales cementantes apartir del Silicoaluminato Cálcico Vítreo (VCAS). Universidad Politécnica de Valencia, Valencia.

Ordóñez, L. M. (2007) Tesis doctoral. Reutilización de la ceniza de cáscara de arroz como material de construcción: Valorización y optimización de sus propiedades puzolánicas. Universidad Politécnica de Valencia, Valencia.

Pérez-Carrión, M. T. (2008) Tesis doctoral. Viabilidad del uso de cenizas de lodo de depuradora en la industria del prefabricado del hormigón. Universidad de Alicante.

Zornoza, E. (2007) Tesis doctoral. El papel del catalizador usado en el craqueo catalítico del petróleo (FCC ó FC3R) como material puzolánico en el proceso de corrosión de las armaduras de hormigón. Universidad Politécnica de Valencia, Valencia.

Zornoza, E., Payá, J., Garcés, P. (2008a) Chloride-induced corrosion of steel embedded in mortars containing fly ash and spent cracking catalyst. Corrosion Science, Vol. 50, pp. 1567-1575.

Zornoza, E., Garcés, P., Payá, J. (2008b) Effect of cement substitution by spent cracking catalyst $(F C 3 R)$ on the corrosion rate of steel rebars embedded in mortars under carbonation attack. Materiales de Construcción, Vol. 58, pp. 27-43.

Zornoza, E., Payá, J., Monzó, J., Borrachero, M. V., Garcés, P. (2009a) The carbonation of OPC mortars partially substituted with spent fluid catalytic cracking catalyst (FC3R) and its influence on their mechanical properties. Construction and Building Materials, Vol. 23, pp. 1323-1328.

Zornoza, E., Garcés, P., Payá, J., Climent, M. A. (2009b) Improvement of the chloride ingress resistance of opc mortars by using spent cracking catalyst. Cement and Concrete Research, Vol. 39, pp. 126-139.

Zornoza, E., Garcés, P., Monzó, J., Borrachero, M. V., Payá, J. (2009c) Accelerated carbonation of cement pastes partially substituted with fluid catalytic cracking catalyst residue. Cement and Concrete Composites, Vol. 31, pp. 134-138.

Zornoza, E., Payá, J., Garcés, P. (2009d) Carbonation rate and reinforcing steel corrosion rate of OPC/FC3R/FA mortars under accelerated condition. Advances in Cement Research, Vol. 21, No. 1, pp. 12-22

¿Es compatible la durabilidad con la sostenibilidad en la industria de la construcción? 